\title{
Mutually Nearest Points for Two Sets in Metric Spaces
}

\author{
Gaj Ram Damai ${ }^{1}$, Prakash Muni Bajracharya ${ }^{2}$ \\ ${ }^{1}$ Department of Mathematics, Tribhuvan University, Siddhnath Science Campus, Mahendranagar, Nepal \\ ${ }^{2}$ Central Department of Mathematics, Tribhuvan University, Kirtipur, Kathmandu, Nepal \\ Email address: \\ gajrajgautam@yahoo.com (G. R. Damai), pmbajracharya13@gmail.com (P. M. Bajracharya)

\section{To cite this article:} \\ Gaj Ram Damai, Prakash Muni Bajracharya. Mutually Nearest Points for Two Sets in Metric Spaces. International Journal of Theoretical \\ and Applied Mathematics. Vol. 4, No. 3, 2018, pp. 29-34. doi: 10.11648/j.ijtam.20180403.11
}

Received: August 27, 2018; Accepted: September 18, 2018; Published: November 7, 2018

\begin{abstract}
In this paper, we give some new conditions for the existence and uniqueness of mutually nearest points of two sets, i.e., two points which achieve the minimum distance between two sets in a metric or Banach spaces. These conditions are seen in case of compact sets, weak Compact sets, closed and convex sets, weakly sequentially compact sets and boundedly compact sets and their combinations. The study is confined to metric spaces and normed or Banach spaces. Some geometric properties of a Banach spaces like; strictly convexity, uniformly convexity, P-Property and weak P-Property are introduced. Also, we introduce the concept of generalized weak P-Property and give some interesting results. The present work may be briefly outlined as follows: It is the mathematical study that is motivated by the desire to seek answers to the following basic questions, among others. Which subsets are mutually proximinal? How does one recognize when given elements $x \in A$ and $y$ $\in B$ are the nearest points of $A$ and $B$ ? which is called a natural extension of the best approximation problem. Can one describe some useful algorithms for actually computing nearest points between two given sets? And how to find closely related sets to the proximity maps.
\end{abstract}

Keywords: Nearest Point, P-Property, Weak P-Property, Compact Set, Minimizing Sequence, Generalized Weak P-Property

\section{Introduction}

The problem of existence and uniqueness of mutually nearest points has been studied by many investigators. In normed linear spaces the conditions under which two sets are distance sets have been studied by Bor-Luh-Lin [15], Dionisio [16], Tukey [17], Klee [18], Pai [19] and others. An application of distance sets to linear inequalities has been cited by Cheney and Goldstein [20]. The same problem in metric spaces has been studied by Nicolescu [21] and is also discussed by Singer [22]. The nearest point and its existence and uniqueness property has been introduced in $[1,2]$ with the use of weak P-Property and generalized weak P- property. A natural extension of the nearest point problem is to find mutually nearest points relative to two sets $A, B$ in a metric space or Best proximity pair evolves as a generalization of the concept of best approximation. Several authors have studied such mutually nearest points. For example; mutually nearest points relative to two closed convex sets in Hilbert spaces are found in many literatures. Mutually nearest points in reflexive Banach spaces is shown by Chong Li [10]. In this paper, the authors have also studied different conditions under which two sets may be distance sets.

\section{Some Definitions and Notations}

Definition 2.1. [4] Let A and B be any two nonempty subsets in a metric space $X$. Then the distance between two sets $A$ and $B$ is defined by

$$
d(A, B)=\inf \{d(x, y): x \in A, y \in B\}
$$

But our Problem is to find the mutual nearest points, i.e., given subsets $\mathrm{A}, \mathrm{B}$ in the metric space $(X, d)$, we find

$(a, b) \in A \times B$ such that

$$
\operatorname{dist}(A, B)=\inf _{x \in A, y \in B} \mathrm{~d}(x, y)=d(a, b)
$$

We call $(a, b)$ a best proximity pair of $A$ and $B$. If there exists the pair $(a, b)$ that satisfies the Equation (1), then we say that $(a, b)$ is the solution of Equation (1). Best approximating pairs in a metric space may not exist in general. If $B$ reduces to a singleton set $\{x\}$ then the Problem (1) reduces to the problem of best approximation when $A \cap B$ 
$\neq \phi$ and it reduces to the well known feasibility problem for two sets and its solution set is

$$
\{(x, x): x \in \mathrm{A} \cap \mathrm{B}\} .
$$

The Formulation (2) captures a wide range of problems in applied mathematics and engineering. It is also used in the solution of linear inequalities, determining separating hyper planes, existence of fixed point and the method of alternating projections[14] etc.

Definition 2.2. Let A, B be nonempty bounded sets in a Banach space $\mathrm{X}$. We define

$$
\begin{gathered}
A=\{x \in A: d(x, y)=d(A, B) \text { for some } y \in B\}, \\
B=\{y \in B: d(x, y)=d(A, B) \text { for some } x \in A\}, \\
(A, B)=\{(a, b) \in A \times B: d(a, \mathrm{~b})=d(A, B)\} .
\end{gathered}
$$

Clearly,

$$
(\mathrm{A}, \mathrm{B}) \subseteq \mathrm{A} \times \mathrm{B}
$$

If there is a pair $(x, y) \in A \times B$ for which

$$
d(x, y)=\operatorname{dist}(A, B)
$$

then the pair $(x, y)$ is called a best proximity pair (mutually nearest points) for $A$ and $B$.

1. If $\mathcal{N}(A, B) \neq \phi$, then we say that $A$ and $B$ are mutual proximinal sets.

2. If $(A, B)$ is singleton, then we call $A, B$ are semiChebyshev sets. If $A$ and $B$ are proximal as well as semiChebyshev, then we call $A$ and $B$ Chebyshev pair.

3. Let $A, B \subseteq X$ be nonempty. Then

i. $\forall u \in X d(A+u, B+u)=\mathrm{d}(A, B)$.

i. $\forall a \in \mathbb{R} d(a A, a B)=|a| d(A, B)$.

iii. $A, B \subseteq X$ are mutually proximinal, iff $A+x$ and $\mathrm{B}+x$ are mutually proximinal for any given $x \in X$.

iv. $A, B \subseteq X$ are mutually proximinal, iff $a A$ and $a B$ are mutually proximinal for any given $a \in \mathbb{R}$.

$$
\text { v. If } z \in X \text {, then } d(A, B) \leq d(z, A)+d(z, B) \text {. }
$$

Theorem 2.3. [6] Let $(X, d)$ be a metric space. Prove that the distance function $d: X \times X \rightarrow \mathbb{R}$ is continuous, assuming. that $X \times X$ has the product topology that results from each copy of $X$ having the topology induced by $d$.

Theorem 2.4 Let $X$ be a metric space, A and B be the subsets of $\mathrm{X}$ and $b \in B$. Then the distance function.

$$
\begin{gathered}
\mathrm{D}_{\mathrm{A}}: \mathrm{B} \rightarrow \mathbb{R}, \text { given by } \\
D_{\mathrm{A}}(b)=d(b, A)(b \in B)
\end{gathered}
$$

is nonexpansive (and so continuous on $A$ ).

Proof. Let $u, v \in B$ and $a \in A$. Then

$$
d(u, A) \leq d(u, a) \leq d(u, v)+d(v, a)
$$

$$
\begin{gathered}
\Rightarrow d(u, A)-d(v, a) \leq d(u, v) \text { for all } a \in A, \text { so } \\
d(u, A)-d(v, A) \leq d(u, v)
\end{gathered}
$$

By symmetry, we have

$$
d(v, A)-d(u, A) \leq d(u, v)
$$

Combining (3) and (4) we get,

$$
|d(u, A)-d(v, A)| \leq d(u, v) .
$$

That is,

$$
\left|\mathrm{D}_{\mathrm{A}}(u)-\mathrm{D}_{\mathrm{A}}(v)\right| \leq d(u, v) .
$$

Hence the distance function DA is non-expansive (and so continuous on A. Indeed, it is uniformly continuous.).

Recall [5] that any sequence $\left(a_{\mathrm{n}}, b_{\mathrm{n}}\right)$ with $\mathrm{a}_{\mathrm{n}} \in \mathrm{A}$ and $\mathrm{b}_{\mathrm{n}} \in B$ for all $\mathrm{n}$ such that $\lim _{\mathrm{n} \rightarrow \infty}\left\|a_{\mathrm{n}}-\mathrm{b}_{\mathrm{n}}\right\|=\mathrm{d}(\mathrm{A}, \mathrm{B})$ is called $\mathrm{a}$ minimizing sequence for $(\mathrm{a}, \mathrm{b}) \in \mathrm{A} \times \mathrm{B}$.

Lemma 2.5 Suppose that $A, B$ are closed subsets of a Banach space $X$. If some minimizing sequence $\left\{\left(x_{\mathrm{n}}, y_{\mathrm{n}}\right)\right\} \subseteq A \times$ $B$ has a weak cluster point $(x, y) \in A \times B$, then

$$
d(A, B)=d(x, y)=\lim _{\mathrm{n} \rightarrow \infty}\left\|x_{\mathrm{n}}-y_{\mathrm{n}}\right\| .
$$

Proof. Let $d(A, B)>0$. We have to prove that

$$
(\mathrm{A}, \mathrm{B}) \subseteq \partial(\mathrm{A}) \times \partial(\mathrm{B}) .
$$

Suppose on the contrary that there exists $(x, y) \in(\mathrm{A}, \mathrm{B})$ such that

$$
(x, y .) \in \operatorname{int} A \times \operatorname{int} B \text {. }
$$

Then

$$
\forall(x, y) \in \mathrm{A} \times \mathrm{B} d(A, B)=\|x-y .\| \leq\|x-y\|
$$

Moreover, there is $\delta \in(., d(x, A))$ such that

$$
B_{X}(x, \delta) \subseteq A \text { and } B_{X}(y, \delta) \subseteq B \text {. }
$$

Put

$$
\begin{array}{r}
x^{\prime}=x_{.}+\delta\left(y_{.}-x_{.}\right) / 2\left\|y_{.}-x .\right\| \in \mathrm{B}_{X}\left(x_{.}, \delta\right) \text { and } \\
y^{\prime}=y_{.}+\delta\left(x_{-} y_{.}\right) / 2\left\|x_{-}-y_{.}\right\| \in \mathrm{B}_{X}\left(y_{.}, \delta\right) .
\end{array}
$$

Then $x^{\prime} \in \mathrm{A}$ and $y^{\prime} \in \mathrm{B}$. Hence

$$
\begin{gathered}
\left\|x^{\prime}-y^{\prime}\right\|=\left\|x+\delta\left(y_{.}-x_{.}\right) / 2\right\| y .-x .\left\|-y_{.}-\delta\left(x_{-}-y_{.}\right) / 2\right\| x_{.}-y .\|\| \\
=\left\|x_{-}-y_{-}-\delta\left(x_{.}-y_{.}\right) /\right\| x_{-}-y .\|\| \\
=\left\|x_{-}-y .\right\|-\delta \\
<\left\|x_{-}-y .\right\| .
\end{gathered}
$$

This contradicts (5) and the proof is complete.

\section{Existence of Mutually Nearest Points}

Theorem 3.1. [9] Let $A$ be a compact set in $\mathbb{R}^{\mathrm{n}}$ and let $B$ be a closed subset of $\mathbb{R}^{\mathrm{n}}$. Define

$$
\mathrm{d}(\mathrm{A}, \mathrm{B})=\inf _{\mathrm{x} \in \mathrm{A}, \mathrm{y} \in \mathrm{B}}\|\mathrm{x}-\mathrm{y}\|
$$


Show that there exist elements $x \in A$ and $y \in B$ such that.

$$
d(A, B)=\|x-y .\|
$$

Proof. The function defined $f(x)=\mathrm{d}(x, B), x \in A$ is continuous and attains its minimum for some $x \in A$, so there exists $y \in B$ such that.

$$
f(x)=\|x-y .\|
$$

Then

$$
d(A, B)=\|x-y .\|
$$

Indeed,

$$
d(x, B) \leq\|x-y\| \text { for all } x \in A \text { and } y \in B .
$$

Thus

$$
f(x) \leq \mathrm{d}(\mathrm{A}, \mathrm{B})
$$

and the reverse inequality,

$$
d(A, B) \leq f(x)
$$

is obvious.

But in this section we will extend this result.

Theorem 3.2. Let $(X, d)$ be a metric space. If $\mathrm{A}$ and $\mathrm{B}$ are compact subsets of $X$, then $(A, B) \neq \phi$.

Proof. The distance function $d: X \times X \rightarrow \mathbb{R}$ is continuous (see Theorem 2.3), and the set $A \times B$ is compact in $X \times X$ by Corollary7.11. Therefore the Extreme Value Theorem applies and indicates that $d$ takes on a minimum value on $A \times B$. That is, there exist $a^{*} \in A$ and $b^{*} \in B$ such that.

$$
\forall \mathrm{a} \in \mathrm{A}, \forall \mathrm{b} \in \mathrm{Bd}\left(\mathrm{a}^{*}, \mathrm{~b}^{*}\right) \leq \mathrm{d}(\mathrm{a}, \mathrm{b}) .
$$

It follows that.

$$
\begin{gathered}
d(A, B)=d\left(a^{*}, b^{*}\right) . \\
\therefore d(A, B) \neq \phi .
\end{gathered}
$$

Proof. By definition of an infimum, we can find a sequence of pairs $\left(\left(u_{\mathrm{n}}, v_{\mathrm{n}}\right)\right)$ in $\mathrm{A} \times \mathrm{B}: d\left(u_{\mathrm{n}}, v_{\mathrm{n}}\right)>a+1 / \mathrm{n}$ for each $n \geq 1$.

Since we are in a metric space, we can use sequential compactness to take subsequences of each sequence and find respective limits $x \in A$ and $y \in B$. Using the triangle inequality, we have for any $\delta>0$

Theorem 3.4. Let $A$ and $B$ be two disjoint compact sets in a metric space $(X, d)$. Then there exists $x \in A$ and $y \in B$ such that $d(x, y)=\inf _{u \in A, v \in B} d(u, v)=a$ (say).

$$
d(x, y) \leq d\left(x, u_{\mathrm{n}}\right)+d\left(u_{\mathrm{n}}, v_{\mathrm{n}}\right)+d\left(v_{\mathrm{n}}, y\right) \leq \delta+\mathrm{a}+1 / \mathrm{n}+\delta .
$$

for all sufficiently large $\mathrm{n}$.

Thus

$$
d(x, y)=a=\inf _{u \in A, v \in B} d(u, v) .
$$

Problem 1. Let $A, B$ be two closed sets ina metric space $X$. Do there exist $\mathrm{a} \in \mathrm{A}$ and $\mathrm{b} \in \mathrm{B}$ such that.

$$
d(a, b)=d(A, B)
$$

1. No, this problem has a negative solution in a general metric space. Indeed, let $X=[-1,0)(0,1]$ be a metric space and $A=(0,1]$. Clearly, $A$ is closed and bounded in $X$. Then for $B=\{-1\}$, the set of distances $\{|x-b|: x \in(0,1], b=-1\}$ has the infimum 1 , but no minimum value.

2. The conclusion does not follow even assuming the completeness of the under-lying metric space. Consider the space $\ell_{\mathrm{p}}$ of sequences (say, $\mathrm{p}=2$ ), and let $A=\left\{a_{\mathrm{n}}\right\}$, where

$$
a_{\mathrm{n}}=(0,0, \cdots, 1+1 / \mathrm{n}, 0,0, \cdots)
$$

Boundedness of $A$ is clear, it is also closed since it has no limit points. However, for $\mathrm{b}=0$, the distance $d\left(b, a_{\mathrm{n}}\right)$ gets arbitrarily close to, but never, 1.

3 . Let $X$ be a metric space endowed with the metric $\rho$ and $F$, $G$ nonvoid, boundedly compact closed sets in $X$. Then there exist elements $f \in \bar{F}$ and $g \in \bar{G}$ such that

This is false. Indeed, let

$$
\rho(f, g .)=\rho(F, G)
$$

$$
\begin{gathered}
F=\left\{(x, y) \in \mathbb{R}^{2}: x \geq 0, y \leq 0\right\}, \\
G=\left\{(x ; y) \in \mathbb{R}^{2}: x>0, y \geq 1 / x\right\} .
\end{gathered}
$$

Then $\mathrm{F}$ and $\mathrm{G}$ are nonvoid boundedly compact sets. But there do not exist $f \in F, g \in G$ satisfying

$$
\begin{gathered}
\rho(f, g .)=\rho(F, G), \\
\because F \cap G=\phi, \rho(F, G)=0 .
\end{gathered}
$$

It is easy to see that the above assertionis true if we add the condition $\mathrm{F}$ or $\mathrm{G}$ is bounded

Theorem 3.5. [4] Let $A$ and $B$ be two non-void sets of a normed linear space X. If $A$ is weakly sequentially compact and $\mathrm{B}$ is convex and proximinal with respect to $A$, then a best proximity pair of $A$ and $B$ exists. For a metric space $X$, we assume instead that $A$ is compact and $B$ is proximinal with respect to $A$.

Theorem 3.6. Let B-A be a compact set in a metric space $\mathrm{X}$. Then for any two compact sets A and B in X

$$
\exists \text { b- a } \in \text { B -A: d(., b - a) =d(., B - A) }
$$

Proof. The distance function $D_{\mathrm{B}-\mathrm{A}}: X \rightarrow \mathbb{R}$ given by

$$
\mathrm{D}_{\mathrm{B}-\mathrm{A}}: p-q \mapsto \mathrm{d}(p-q) \quad(\text { where } p-q \text { in } B-A)
$$

is continuous, and by assumption the set $\mathrm{B}$ - A is compact. Thus the Extreme Value Theorem applies and indicates that $\mathrm{D}_{\mathrm{B}-\mathrm{A}}$ takes on a minimum value on $B-A$. That is, there exist $\mathrm{b}-\mathrm{a} \in B-A$ such that

$$
\begin{aligned}
d(0, b-a)=\inf & \{d(0, p-q): p-q \in B-A\} \\
= & d(0, B-A \\
= & d(A, B)
\end{aligned}
$$

Theorem 3.7 Let $A$ and $B$ be any two nonempty closed 
subsets in a metric space, (X, d). If $\left\{x_{\mathrm{n}}\right\} \subseteq \mathrm{A}$ has a limit point $u$ in $A$ and $\left\{y_{\mathrm{n}}\right\} \subseteq \mathrm{B}$ has a limit point $v$ in $B$. Then

$$
d(u, v)=d(A, B)
$$

Proof. Let $\left\{x_{\mathrm{n}}\right\} \subseteq A$ be a minimizing sequence for $x$, i.e.,

$$
d\left(x, x_{\mathrm{n}}\right) \rightarrow d(x, A) .
$$

Since $A$ is closed it has a limit point $u \in A$, then passing to a subsequence we may assumethat $d\left(u, x_{\mathrm{n}}\right) \rightarrow$.

We then have

$$
d(x, A) \leq d(x, u) \leq d\left(x, x_{\mathrm{n}}\right)+d\left(x_{\mathrm{n}}, z\right) \rightarrow d(x, A)=d(x, A) .
$$

This implies that

$$
\mathrm{d}(\mathrm{x}, \mathrm{u})=\mathrm{d}(\mathrm{x}, \mathrm{A}) \text {. }
$$

In the same vein if $\left\{y_{\mathrm{n}}\right\} \subseteq B$ be a minimizing sequence for $v$ in $B$, then it has a limit point $v$ in $B$ and

$$
d(x, v)=d(x, B) \text {. }
$$

We then have

$$
\begin{gathered}
\mathrm{d}(\mathrm{A}, \mathrm{B}) \leq \mathrm{d}(\mathrm{u}, \mathrm{v}) \leq \mathrm{d}(\mathrm{u}, \mathrm{x})+\mathrm{d}(\mathrm{x}, \mathrm{v}) \\
=\mathrm{d}(\mathrm{x}, \mathrm{A})+\mathrm{d}(\mathrm{x}, \mathrm{B}) \\
=\mathrm{d}(\mathrm{A}, \mathrm{B}) \\
\Rightarrow \mathrm{d}(\mathrm{u}, \mathrm{v})=\mathrm{d}(\mathrm{A}, \mathrm{B}) .
\end{gathered}
$$

Corollary 3.8 [4] Let $X$ be a reflexive Banach space and $A$, $B$ two nonvoid subsets of $X$. If $\mathrm{A}$ is bounded, weakly closed, and $B$ is closed convex, then best proximity pair of $A$ and $B$ exists.

Indeed, any bounded, weakly closed subset of a reflexive space $X$ is weakly sequentially compact. Since any closed convex subset is proximinal with respect to the total space (it follows from above theorem). Obviously, a compact set is a weakly sequentially compactset. A boundedly compact set in metric space and a closed convex set in a uniformly convex Banach space are all proximinal with respect to the total space.

\section{Uniqueness of Mutually Nearest Points}

For the uniqueness of the proximal pair, we have to impose an additional condition on $X$. In general, if $A$ and $B$ are closed and one of them is compact, the proximal pair may not exist for arbitray normed space $X$. However, if $X$ is uniformly convex, then the proximal pair exists.

Theorem 4.1.[11] Let $X$ be a uniformly convex Banach space and $A, B$ be two closed convex sets such that one of them is compact. Then the proximal points a, b of $A$ and $B$ exist and they are unique if

$$
(A-A) \backslash(B-B)=\{0\}
$$

Theorem 4.2. [5] Let $S$ and $W$ be two strictly convex subsets of X. Then $\mathcal{N}(S, W)$ contains at most one element.
Proof. Suppose that

$$
(s, w),\left(s_{1}, w_{1}\right) \in \mathcal{N}(S, W)
$$

Then

$$
d(S, W)=\|s .-w .\|=\left\|s_{1}-w_{1}\right\|
$$

and

$$
\forall(s, w) \in S \times W d(S, W) \leq\|s-w\|
$$

It follows from (8) and (9) that $w \in \mathrm{P}_{W}(s), w_{1} \in \mathrm{P}_{W}\left(s_{1}\right)$.

Thus, Lemma 2.5 guarantees that

$$
\left\{w, w_{1} \subset \partial \mathrm{W} .\right.
$$

Below we prove

$$
w=w_{1}
$$

Since $\mathrm{W}$ is strictly convex, it suffices to showthat

$$
\exists \lambda \in(0,1)(1-\lambda) w+\lambda w_{1} \in \partial W .
$$

To do this, let $\lambda \in(0,1)$. Then, by the convexity of $S$ and $\mathrm{W}$, one has

$$
\begin{gathered}
(1-\lambda) s+\lambda s_{1} \in S . \\
(1-\lambda) w+\lambda w_{1} \in W .
\end{gathered}
$$

This together with (3) and (4) implies that

$$
\begin{gathered}
d(S, W) \leq\left\|(1-\lambda) s_{+}+\lambda s_{1}-\left[(1-\lambda) w+\lambda w_{1}\right]\right\| \\
\leq(1-\lambda)\left\|s_{-}-w .\right\|+\lambda\left\|s_{1}-w_{1}\right\| \\
=d(S, W)
\end{gathered}
$$

It follows from (5) and (6) that for all $w \in W$.

$$
\begin{gathered}
\left\|(1-\lambda) \mathrm{s}+\lambda \mathrm{s} 1-\left[(1-\lambda) \mathrm{w}+\lambda \mathrm{w}_{1}\right]\right\| \\
\leq \lambda\left\|(1-\lambda) \mathrm{s}+\lambda \mathrm{s}_{1}-\mathrm{w}\right\|
\end{gathered}
$$

which means that $(1-\lambda) w+\lambda w_{1} \in \mathrm{P}_{\mathrm{w}}\left((1-\lambda) s+\lambda s_{1}\right)$,

$$
(1-\lambda) w+\lambda w_{1} \in \partial \mathrm{W} \text {. }
$$

by Lemma 2.5 as desired. Similarly, we can prove that $s .=s_{1}$. Consequently,

$$
\left(s_{.}, w .\right)=\left(s_{1}, w_{1}\right)
$$

This completes the proof.

Definition 4.3. [5] Recall that a nonemptysubset A of $X$ is called strictly convex if, for any two distinct points $x, y \in \partial \mathrm{A}$ and $\alpha \in(0,1)$, one has that

$$
\alpha x+(1-\alpha) y \in \mathrm{A} .
$$

$X$ is called strictly convex if $B(X)$ is strictly convex.

Remark 4.4. If we only assume that one of $S$ and $W$ is strictly convex, the above theorem does not hold in general. However, if $X$ is strictly convex, then the previous theorem 
can be improved to the following results.

Theorem 4.5. [5] Let $X$ be strictly convex. Let one of $S$ and $W$ be also strictly convex and the other be quasi-convex. Then $\mathcal{N}(S, W)$ contains at most an element.

Theorem 4.6. [5] Let $X$ be strictly convex and smooth. Let one of $S$ and $W$ be also strictly convex while the other be a sun. Then $\mathcal{N}(S, W)$ contains at most an element.

Remark 4.7. In Theorem 4.5 (resp. Theorem4.6), if one of $S$ and $W$ is not quasi-convex(resp. a sun), then the conclusion is not true in general.

Definition 4.8. [3] A pair $(A, B)$ of nonempty of a Banach space $X$ is said to have P-Property if

$$
\begin{gathered}
\mathrm{A} \neq \phi \forall \mathrm{x}, \mathrm{y} \in \mathrm{A} \text { and } \mathrm{u}, \mathrm{v} \in \mathrm{B} . \\
\|x-u\|=\|y-v\|=\mathrm{d}(A, B) \Rightarrow\|x-y\|=\|u-v\| .
\end{gathered}
$$

Definition 4.9. [3] A Banach space $X$ is said to have $\mathrm{P}$ Property if every pair $(A, B)$ of nonempty closed convex subsets of $\mathrm{X}$ has P-Property.

Theorem 4.1. Let $A, B$ be non-empty setsin a metric space $X$. If $A$ is compact and $B$ is closed in $X$ and $X$ has $P$-property, then

$$
\exists ! \mathrm{a} \in A \exists \mathrm{b} \in B: \mathrm{d}(\mathrm{a}, \mathrm{b})=\mathrm{d}(A, B) .
$$

Proof. In Theorem 3.6, we have shown that

Suppose that

$$
\exists \mathrm{a} \in A \exists \mathrm{b} \in B: \mathrm{d}(\mathrm{a}, \mathrm{b})=\mathrm{d}(A, B) .
$$

For the uniqueness, we observe that $A=\{x\}$ and $B$ are closed sets in $X$. Since $X$ has P-Property we have

$$
\mathrm{d}(\mathrm{a}, \mathrm{b})=\mathrm{d}(\mathrm{u}, \mathrm{v})=\operatorname{dist}(\mathrm{A}, \mathrm{B}) \Rightarrow \mathrm{d}(\mathrm{a}, \mathrm{u})=\mathrm{d}(\mathrm{b}, \mathrm{v}) .
$$

Taking $\mathrm{A}=\{\mathrm{x}\}, \mathrm{B}$ as it is with $u, v \in B$ in the definition of P-Property. We get,

$$
d(u, v)=0 \Rightarrow u=v .
$$

The uniqueness result can be obtained under more weaker condition on $\mathrm{X}$.

Definition 4.11. [7] Let $(A, B)$ be a pair of nonempty closed subsets of a metric $\operatorname{space}(X, d)$ with $A \neq \phi$. The pair $(A, B)$ is said to have weak $\mathrm{P}$ - property if

$$
d(x, u)=d(y, v)=\operatorname{dist}(A, B) \Rightarrow \mathrm{d}(x, y) \leq d(u, v) .
$$

where $x, y \in A$. and $u, v \in B$.

Theorem 4.12. [8] Let $A$ and $B$ be two sets in a metric space $X$ and $x \in A$. If $B$ is compact and $X$ has weak $P$-property then

$$
\exists ! \mathrm{a} \in A \exists \mathrm{b} \in B: \mathrm{d}(\mathrm{a}, \mathrm{b})=\mathrm{d}(A, B) .
$$

Proof. In Theorem 3.6, we have shown that

$$
\exists v \in B: d(x, v)=d(x, B) .
$$

For the uniqueness, we observe that $B$ and $\{x\}$ are closed sets in $X$. Since $X$ has weak $P$ - property, we have

$$
d(x, u)=d(x, v)=\operatorname{dist}(A, B) \Rightarrow d(u, v) \leq \mathrm{d}(x, x)=0 .
$$

Taking $B$ as it is and $\mathrm{A}=\{x\}$ with $u, v \in B$ in the definition of weak P-property, we get

$$
d(u, v)=0 \Rightarrow u=v
$$

Definition 4.13. [2] A pair $(A, B)$ of nonempty subset of a Banach space $X$ is said to have generalized weak $P$-property if

$$
\begin{gathered}
A \neq \phi, \forall x, y \in A \text { and } u, v \in \mathrm{B} . \\
\|x-\mathrm{u}\|=\|\mathrm{y}-\mathrm{v}\|=d(\mathrm{~A}, \mathrm{~B}) \Rightarrow\|\mathrm{x}-\mathrm{y}\| \leq \alpha\|\mathrm{u}-\mathrm{v}\|, \alpha>1 .
\end{gathered}
$$

Theorem 4.14. Let $A$ and $B$ be two sets in a metric space $X$ and $x \in A$. If $B$ is compact and $\mathrm{X}$ has generalized weak $P$ property then

$$
\exists ! \mathrm{a} \in A \exists \mathrm{b} \in B: \mathrm{d}(\mathrm{a}, \mathrm{b})=\mathrm{d}(A, B) .
$$

Proof. In Theorem 3.6, we have shown that

$$
\exists v \in B: d(x, v)=d(x, B) .
$$

For the uniqueness, we observe that $B$ and $\{x\}$ are closed sets in $X$. Since $X$ has generalized weak $P$-property we have

$$
d(x, u)=d(x, v)=\operatorname{dist}(A, B)) d(\mathrm{u}, v) \leq \alpha d(x, x)=0,
$$

taking $B$ as it is and $A=\{x\}$ with $\mathrm{u}, v \in B$ in the definition of generalized weak $P$-property we get

$$
d(u, v)=0 \text {. This implies that } u=v
$$

\section{Characterization of Proximal Points}

The characterization of proximal points for pairs of sets, not necessarily convex is discussed in [12] by employing "solar properties" of sets also discussed in [11]. Moreover, some duality results for the distance $d(U, V)$ are also dealt with there.

Theorem 5.1. [12] Let $X$ be a normed linear space whose dual $X^{*}$ is strictly convex. Then $u \in U, v \in V$ are proximal if and only if $\mathrm{u}$ is a nearest point of $\mathrm{v}$ in $U$ and $\mathrm{v}$ is a nearest point of $\mathrm{u}$ in $\mathrm{V}$. The characterization of proximal points are

Theorem 5.2[13] Let $U, V$ be convex sets. Then $\bar{u} \in U$, $\bar{v} \in V$ are proximal if and only if there exists an $\mathrm{f} \in \mathrm{X}^{*}$ such that

(1) $\|f\|=1$,

(2) $\operatorname{Re} f(u-\bar{u}) \geq 0$ for each $u \in U$,

(3) $\operatorname{Re} f(v-\bar{v}) \leq 0$ for each $v \in V$,

(4) $f(\bar{u}-\bar{v})=\|\bar{u}-\bar{v}\|$.

\section{Conclusion}

The mutually nearest points for two sets in a given space $X$ has been an active topic of research for several years. In this paper, some of its more ramifications are discussed. We add the different conditions on the subsets $A$ and $B$ in $X$. We also incorporated some geometrical property of the given space $X$ so that there exists the unique mutually nearest points. A new geometrical property called generalized weak $P$ - property of 
a given space $X$ is introduced and we use it for the existence and uniqueness of mutually nearest points. Finally, the characterizations of mutually nearest points are also seen. So this topic is a fertile field in the garden of many branches of mathematics.

\section{Acknowledgements}

I would like to express my gratitude to my $\mathrm{Ph} . \mathrm{D}$ advisor, Prof. Prakash Muni Bajracharya and my mentors for their encouragement, constant support, helpful remarks and insightful comments on the paper and I also would like to express my deep gratitude to UGC of Nepal for financial support.

\section{References}

[1] P. M. Bajracharya, G. R. Damai, "A note on existence and uniqueness of nearest points in a set", International Journal of Research and Review, (2018), 5(1), 5.-54.

[2] P. M. Bajracharya and G. R. Damai, "On Nearest Points of a Set", GIJASH, (2018) Vol.2, Issue 3, 5-8.

[3] V. R. Sankar and A. A. Eldred, "Characterization of Strictly Convex Spaces and Applications," Journal of Optimization Theory and Applications, (2014) Volume 160, No.2, 703-710.

[4] Xiubin Xu, "A Result on Best Proximity Pair of Two Sets", Journal of Approximation Theory, (1988)54, 322-325.

[5] Xianfa Luo, "Characterizations and Uniqueness of Mutually Nearest Pointsfor Two Sets in Normed Spaces, "Numerical Functional Analysis and Optimization, (2014)35(5), 611-622.

[6] C. C. Adams and R. D. Fran-zosa, "Introduction to topology Pure and applied," Upper Saddle River, NJ, Pearson Prentice Hall, (2008).

[7] J. Zhang, Y. Su, Q. Cheng, "A note on a best proximity point theorem for Geraghty contractions," Fixed Point Theory Appl., 2.13, 99 (2013).

[8] V. R. Shankar, "A best proximity point theorem for weakly contractive non-self mappings," Nonlinear Anal. (2011)74, 4804-4808.
[9] R. Shakarchi, "Problems and Solutions for Undergraduate Analysis," Library of Congress Cataloging in-Publication Data (1998), ISBN 0-387-98235-3.

[10] Chong Li, "On Mutually Nearest and Mutually Furthest Points in Reflexive Banach Spaces," Journal of Approximation Theory, (2000)103, 1-17.

[11] D. V. Pai, "Proximal Points of Convex Sets in normed Linear Spaces," Yokohama Math., (1974)9, 53-78.

[12] D. V. Pai and H. G. ter Morsche, "On proximal points of pairs of sets," T. H.-Report 75-WSK-OI January (1975).

[13] D. V. Pai, "A Characterization of Smooth Normed Linear Spaces," Journal of Approximation Theory, (1976)17, 315-32.

[14] W. K. Gunt, "An alternating projections method for certain linear problems in a Hilbert space," IMA Journal of Numerical Analysis (1995) 15, 291-305.

[15] Lin-Bor-Luh, "Distance-sets in normed vector spaces". Nieuw Arch. Wiskunde, (1966) (3)14, 23-3.

[16] J. J. Dionisio, "On the separation of convex sets in R". Univ. Lisboa Revista Fae., Ci. (A 2), 1.(1963\64), 185-94.

[17] J. W. Tukey, "Some notes on the separation of convex sets," Portugaliae Math., (1942)3, 95-1.2.

[18] V. L. Klee, "Separaticn properties of convex sets,"Proe. Am. math. Soc., (1955)6, 313-17.

[19] D. V. Pai, "Proximinal points of convex sets in normed linear spaces,"Research Report, 1.1. T., Bombay. th. Soc., 6, 3(1972), 13-17.

[20] W. Cheney, and A. A. Goldstein, "Proximity maps for convex sets,"Proc. Am. math. Soc.,(1969)1., 448-5.

[21] M. Nicolescu, "Sur la meilleure approximation d'une fonction donnee par les functions d'une famille donnee,"But. Fae. Sfi., Cern; Uli,(1938)12, 120-280.

[22] I. Singer, "Best Approximation in Normed Linear by Elements of Linear Subs paces," Springer-Verlag, Berlin-Heideiberg, (1970). 